\title{
Climate variability and dengue fever in Makassar, Indonesia: Bayesian spatio-temporal modelling
}

\author{
Aswi Aswi ${ }^{\mathrm{a}, \mathrm{b}, \mathrm{d}}$, Susanna Cramb ${ }^{\mathrm{a}, \mathrm{c}}$, Earl Duncan ${ }^{\mathrm{a}, \mathrm{d}}$, Wenbiao $\mathrm{Hu}^{\mathrm{c}}$, Gentry White ${ }^{\mathrm{a}, \mathrm{d}}$, \\ Kerrie Mengersen ${ }^{\mathrm{a}, \mathrm{d}, *}$ \\ ${ }^{a}$ ARC Centre of Excellence for Mathematical and Statistical Frontiers, Queensland University of Technology, Australia \\ ${ }^{\mathrm{b}}$ Universitas Negeri Makassar, Indonesia \\ ' School of Public Health and Social Work, Queensland University of Technology, Australia \\ d Science and Engineering Faculty, School of Mathematical Science, Queensland University of Technology, GPO Box 2434, Brisbane, Queensland 4001, \\ Australia
}

\section{A R T I C L E I N F O}

\section{Article history:}

Received 10 June 2019

Revised 10 November 2019

Accepted 4 December 2019

Available online 7 February 2020

\section{Keywords:}

Bayesian

Spatio-temporal conditional autoregressive

Climatic factors

\begin{abstract}
A B S T R A C T
A range of Bayesian models have been used to describe spatial and temporal patterns of disease in areal unit data. In this study, we applied two Bayesian spatio-temporal conditional autoregressive (ST CAR) models, one of which allows discontinuities in risk between neighbouring areas (creating 'groups'), to examine dengue fever patterns. Data on annual (2002-2017) and monthly (January 2013 - December 2017) dengue cases and climatic factors over 14 geographic areas were obtained for Makassar, Indonesia. Combinations of covariates and model formulations were compared considering credible intervals, overall goodness of fit, and the grouping structure. For annual data, an ST CAR localised model incorporating average humidity provided the best fit, while for monthly data, a single-group ST CAR autoregressive model incorporating rainfall and average humidity was preferred. Using appropriate Bayesian spatio-temporal models enables identification of different groups of areas and the impact of climatic covariates which may help inform policy decisions.
\end{abstract}

Crown Copyright @ 2020 Published by Elsevier Ltd. All rights reserved.

\section{Introduction}

Dengue fever (DF) affects more than one hundred million people every year and is one of the most important mosquito-borne diseases in the world (Vanessa et al., 2012). DF is the main source of human deaths from vector borne disease (Gubler, 1998). The dengue viruses are transmitted by the female mosquito Aedes aegypti, whose breeding and development are influenced by climatic factors such as rainfall, humidity, and temperature (Yu et al., 2016). Incubation within mosquitoes ranges from 8 to 12 days, and within humans from 3 to 14 days before symptoms appear (Chan and Johansson, 2012).

When examining small areas within a country, Bayesian disease mapping approaches are often useful. Bayesian spatio-temporal (ST) models enable the time-varying nature of climatic variables and dengue fever to be captured over small areas. Often the spatial random effects in these models assume a conditional autoregressive (CAR) prior. This prior can be formulated in a number of ways.

\footnotetext{
* Corresponding author at: Science and Engineering Faculty, School of Mathematical Sciences, Queensland University of Technology, GPO Box 2434, Brisbane, Queensland 4001, Australia.

E-mail address: k.mengersen@qut.edu.au (K. Mengersen).
}

Two popular priors are the Besag et al. (1991), and the Leroux et al. (2000), described in more detail below. Both these models allow for a combination of smoothing over nearby areas as well as towards the overall average. The BYM and Leroux models have been criticised by some authors for the potential to oversmooth the risks when there are genuine differences between neighbouring areas (Lee and Mitchell, 2013; Rodrigues and Assunção, 2012). Hence, it would be beneficial to have a model that allows for spatial autocorrelation between adjacent areas within discontinuous groups.

Recently, several forms of Bayesian ST models have been introduced which allow for such discontinuities. Lee and Lawson (2016) introduced a Bayesian ST CAR localised model which separates areas into different groups, and only smooths over adjacent areas if they are within the same group. They compared three Bayesian ST models: ST CAR localised model (Lee and Lawson, 2016), ST CAR ANOVA model (Knorr-Held, 1999), and ST CAR autoregressive (AR) model (Rushworth et al., 2014), for describing maternal smoking rates with and without socio-economic covariates. The authors found that the ST CAR localised model outperformed those which did not allow for discontinuities (ST CAR ANOVA and ST CAR AR) in terms of WAIC. However, to our knowledge, this class of models has not been used in examining dengue fever and climatic influences. 
This paper aims to determine the most appropriate Bayesian model and corresponding spatial priors for data collected at different temporal resolutions (monthly and annually) in order to detect spatio-temporal patterns of dengue fever. The impact of covariates on the groups identified in an ST CAR localised model is also evaluated, in order to assess the importance of climatic variables on dengue transmission across Makassar. The structure of the ST CAR localised model is the same as an ST CAR AR, but there is an additional grouping component in the localised model which separates areas into groups and random effects are modelled with an intrinsic CAR (ICAR) prior, described below. We also compare the ST CAR AR and ST CAR localised models when there is only one group identified.

\section{Methods and materials}

\subsection{Study area}

Dengue fever mostly affects sub-tropical and tropical areas. It is endemic to Indonesia which had the highest number of cases in the Southeast Asia region in 2010 (Chan, 2012). The average number of cases during 2000 to 2011 was 94,647 cases, with deaths per year ranging from 472 to 1446 (WHO-SEARO, 2011). Makassar, the capital city of South Sulawesi Province, is the fifth largest city in Indonesia according to population, with a population in 2017 of approximately 1.5 million (737,146 males and 751,865 females). The city's area is $175.79 \mathrm{~km}^{2}$, and it has 14 administrative districts with populations ranging from 28,696 to 208,436 in 2017. The population density of Makassar city was 8471 people per kilometre square in 2017, and the average number of residents per household was about four people (BPS, 2018). Makassar district is the city centre of Makassar. Mariso, Mamajang, and Makassar districts are urban areas; Biringkanaya, Tamalanrea, and Manggala districts are rural areas, and the remaining districts are suburban areas (see Fig. 1). Tamalanrea and Makassar district had the lowest and the highest population densities, respectively. Tamalate, Rappocini, and Makassar districts have a number of areas that are categorised as slums. Biringkanaya is the largest district in Makassar city but only one subdistrict is categorised as a slum area.

\subsection{Dengue data}

The number of dengue cases was obtained from the Makassar city Health Office, South Sulawesi Province, Indonesia covering annual time periods from 2002 to 2017 by district. The monthly dengue fever incidence from January 2013 to December 2017 was also obtained by district and sex. An ethics exemption (exemption number: 1700000479) was obtained from Queensland University of Technology (QUT) as the count data were non-identifiable.

\subsection{Climatic variables}

Indonesia has two seasons, the rainy season from November to April and the dry season from May to October. The temperature is relatively constant throughout the year, ranging from 23 to 34 'Celsius. Climatic variables were gathered from the Meteorology, Climatology, and Geophysical Agency from January 2002 to December 2017 (htttp://dataonline.bmkg.go.id/home). These variables were daily minimum temperature, maximum temperature, mean temperature, mean humidity, and the amount of rainfall. Due to collinearity issues, only one of the temperature variables was included in any combination of covariates used in modelling.

Makassar has four rainfall stations: BBMKG region IV Panaikang station in Panakkukang (rainfall station 1); Biring Romang station in Manggala (rainfall station 2); BPP Barombong station in Tamalate (rainfall station 3); and Maritime meteorology station
Paotere in Ujung Tanah (rainfall station 4). The rainfall for each district is taken from the nearest rainfall station. This was defined based on distance to district centroids using the R package fields, function rdist in R version 3.3.3 (R Core Team, 2019); see Fig. 1. The amount of monthly precipitation fluctuates, ranging from $0 \mathrm{~mm}$ to $1054.2 \mathrm{~mm}$. There are some missing monthly precipitation values in the dataset $(17 / 768=2.2 \%)$. These missing values were estimated by taking the average of non-missing neighbouring time series values in time for the corresponding rainfall station.

As the climatic variables scales differs significantly, all climatic covariates are standardised to have zero mean and standard deviation of unity for numerical stability, to equalise the data variability and range.

\subsection{Model formulation}

Two ST CAR models were used to assess the presence of groups and importance of climatic variables on dengue transmission across Makassar.

\subsubsection{Spatio temporal CAR localised model}

This model proposed by Lee and Lawson (2016) is capable of identifying groups of areas that display different values of the response compared with their geographical and temporal neighbours. It is therefore not restricted by the assumption that two areas that are close together should have similar responses (after accounting for specified covariates). The model assumes a Poisson distribution for the response and is formulated as follows,

$y_{i j} \sim \operatorname{Poisson}\left(e_{i j} \theta_{i j}\right)$

$\log \left(\theta_{i j}\right)=\mathrm{x}_{i j}^{T} \alpha+u_{i j}+\lambda_{Z_{i j}}$

where $y_{i j}$ is the observed number of dengue cases in the $i$ th area and $j$ th time period, $i=1, \ldots, I ; j=1, \ldots, J$; and $e_{i j}$ and $\theta_{i j}$ are the expected number of dengue cases and the relative risk of dengue respectively, each in area $i$ at time $j$. The expected number of dengue cases $\left(e_{i j}\right)$ was calculated as the overall incidence rate for the entire Makassar region over the entire time period multiplied by the population at risk in each area and time period, $e_{i j}=\frac{\sum_{i} \sum_{j} y_{i j}}{\sum_{i} \sum_{j} p_{i j} p_{i j}}$ pop $_{i j}$. The dengue raw standardised incidence ratio (SIR) (i.e. the relative risk) was calculated as the ratio of the observed number of dengue cases to the expected number of cases.

In the above model, $x_{i j}^{T} \alpha$ represent a linear combination of the selected covariates (fixed effects), and the two sets of latent components, $u_{i j}$ and $\lambda_{z_{i j}}$ are smoothing components, a spatially and temporally autocorrelated variation and a piecewise constant grouping or intercept component, respectively. Spatially and temporally adjacent data points $\left(y_{i j}, y_{k l}\right)$ will be autocorrelated if $\lambda_{z_{i j}}=\lambda_{z_{k l}}$ (the same intercept), but display very different values if $\lambda_{z_{i j}} \neq \lambda_{z_{k l}}$. In the context of dengue fever incidence, $\lambda_{z_{i j}} \neq \lambda_{z_{k l}}$ allows spatially and temporally neighbouring areas to have very different probabilities of dengue fever. This piecewise intercept component $\lambda_{Z_{i j}}$ can therefore identify distinct groups of areas with high or low probabilities of dengue disease. This encompasses at most $G\left(\lambda_{1}<\lambda_{2}<\lambda_{3}<\cdots<\lambda_{G}\right)$ distinct groups which are ordered through the prior:

$\lambda_{\mathrm{k}} \sim$ Uniform $\left(\lambda_{\mathrm{k}-1}, \lambda_{\mathrm{k}+1}\right)$ for $k=1,2, \ldots, G$

where $\lambda_{0}=-\infty$ and $\lambda_{G+1}=+\infty$. An observation in area $i$ at time $j$ is allocated to one of the $G$ intercepts by $Z_{i j} \in\{1,2,3, \ldots, G\}$. The value of $G$ is fixed in the model and it is recommended to set it to a small odd number (Lee and Sarran, 2015). In the case study below, values of $G=2$ and $G=3$ are considered. Larger values are not considered because of the small number of areas. The value of 


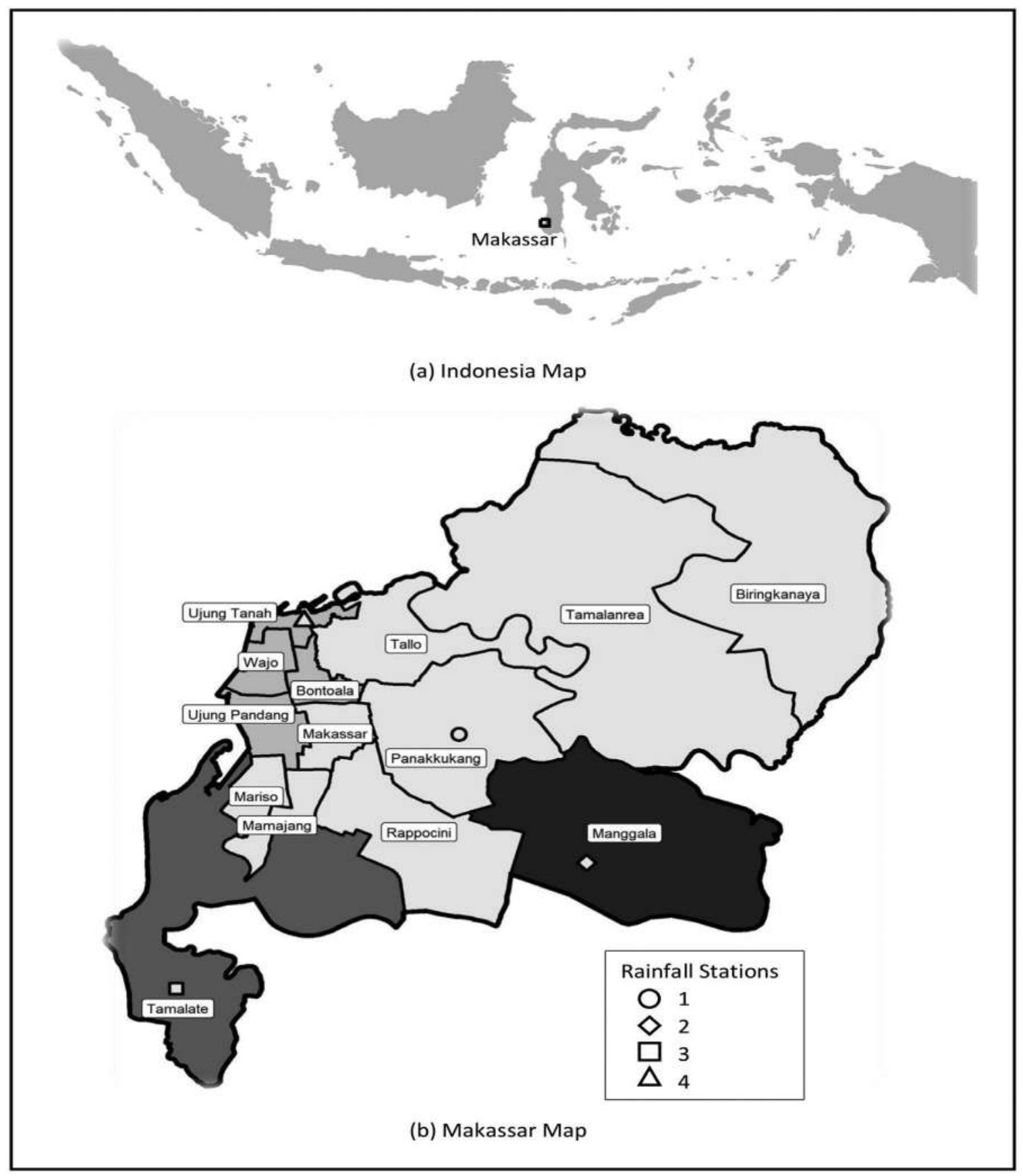

Fig. 1. Indonesia map (a) and Makassar map showing the 14 districts and the location and coverage of the four rainfall stations (b).

$Z_{i j}$ is penalised towards the middle intercept value $G^{*}: G^{*}=\frac{G+1}{2}$ if $G$ is odd and $G^{*}=\frac{G}{2}$ if $G$ is even through a penalty function $f\left(Z_{i j}\right)$ :

$$
\begin{aligned}
f\left(Z_{i j} \mid Z_{i, j-1}\right)= & \frac{\exp \left(-\delta\left[\left(Z_{i j}-Z_{i, j-1}\right)^{2}+\left(Z_{i j}-G^{*}\right)^{2}\right]\right)}{\sum_{r=1}^{G} \exp \left(-\delta\left[\left(r-Z_{i, j-1}\right)^{2}+\left(r-G^{*}\right)^{2}\right]\right)} \\
& \text { for } j=2, \ldots, J,
\end{aligned}
$$$$
f\left(Z_{i 1}\right)=\frac{\exp \left(-\delta\left(Z_{i 1}-G^{*}\right)^{2}\right)}{\sum_{r=1}^{G} \exp \left(-\delta\left(r-G^{*}\right)^{2}\right)}
$$

where $\delta$ is the penalty parameter, $\delta \sim \operatorname{Uniform}\left(\mathrm{L}_{\delta}, \mathrm{U}_{\delta}\right)$. In the case study below, the hyperparameters $\mathrm{L}_{\delta}$ and $\mathrm{U}_{\delta}$ are set to 1 and 100 , respectively, in keeping with published advice (Lee and Lawson, 2016).

The smoothing components $u_{i j}$ are modelled via the multivariate autoregressive process with spatial autocorrelation $\rho_{S}=1$ which corresponds to an ICAR model.

$u_{1} \sim \mathrm{N}\left(\mathbf{0}, \tau^{2} \mathbf{Q}\left(\mathbf{W}, \rho_{S}\right)^{-1}\right)$,

$\left(u_{j} \mid u_{j-1},\right) \sim \mathrm{N}\left(\rho_{T} u_{j-1}, \tau^{2} \mathbf{Q}\left(\mathbf{W}, \rho_{S}\right)^{-1}\right) \quad j=2, \ldots J$,

$\tau^{2} \sim$ Inverse - Gamma $(1,0.01)$ 
$\rho_{T} \sim \operatorname{Uniform}(0,1)$

These values were set for the case study in keeping with published advice (Lee and Lawson, 2016). A value of $\rho_{S}=1$ imposes strong spatial smoothing on $u_{j}$, so that any different values (step changes) in the surface are controlled instead by $\lambda_{z_{i j}}$.

\subsubsection{Spatio temporal CAR AR model}

The second model we use is the ST CAR AR model described by Rushworth et al. (2014). This model structure has some similarities to the ST localised model, but the linear predictor has no group component $\lambda$ and random effects $u_{i j}$ are modelled with varying $\rho_{S}$, allowing for additional unstructured as well as spatially structured smoothing. The model is given by:

$y_{i j} \sim \operatorname{Poisson}\left(e_{i j} \theta_{i j}\right)$

$\log \left(\theta_{i j}\right)=\mathrm{x}_{i j}^{T} \alpha+u_{i j}$

where these terms are defined as before, except here $u_{i j}$ differs slightly, i.e.

$u_{1} \sim \mathrm{N}\left(\mathbf{0}, \tau^{2} \mathbf{Q}\left(\mathbf{W}, \rho_{S}\right)^{-1}\right)$,

$\left(u_{j} \mid u_{j-1}\right) \sim \mathrm{N}\left(\rho_{T} u_{j-1}, \tau^{2} \mathbf{Q}\left(\mathbf{W}, \rho_{S}\right)^{-1}\right) \quad j=2, \ldots, J$,

In this model, the spatial surface at time $j, u_{j}=\left(u_{1 j}, \ldots, u_{I j}\right)$, is the vector of random effects for time period $j$, which evolves over time through a first order autoregressive AR(1) process with temporal autoregressive parameter $\rho_{T}$.

The precision matrix $\mathbf{Q}\left(\mathbf{W}, \rho_{S}\right)=\rho_{S}[\operatorname{diag}(\mathbf{W} 1)-\mathbf{W}]+$ $\left.\left(1-\rho_{S}\right) \mathbf{I}\right)$ corresponds to the CAR Leroux prior (Leroux et al., 2000) where $I$ and $\mathbf{1}$ are the $I \times I$ identity matrix and the $I \times 1$ vector of ones respectively. Weakly informative inverse-gamma priors are used for the variance, while Uniform and Gaussian priors are used for the dependence and regression parameters respectively:

$\tau^{2} \sim$ Inverse - Gamma $(1,0.01)$

$\rho_{S}, \rho_{T} \sim \operatorname{Uniform}(0,1)$

$\alpha \sim N(\mathbf{0}, 1000 \mathbf{I})$

We used a set of model combinations to investigate the most appropriate spatial priors and the impact of covariates on the group identified at different temporal resolutions. We compared different versions of the ST CAR localised model allowing a maximum of two $(G=2)$ and three $(G=3)$ groups, without climatic covariates and then with different combinations of climatic covariates. We considered each of the main climatic variables separately (average humidity, average temperature, and rainfall), then examined combinations of two then three, together (average humidity, rainfall and one of the temperature variables). In total, we had 9 different combinations of models run for both $G=2$ and $G=3$.

Parameters of models were estimated using the CARBayesST package version 2.5.1 in R version 3.3.3 (R Core Team, 2019). Posterior quantities for parameters were based on 100,000 MCMC samples collected after a burn-in of 20,000 samples.

Different combinations of covariates and spatio-temporal model formulations are compared with respect to three metrics: whether the $95 \%$ posterior credible interval $(\mathrm{CI})$ for the climatic covariate coefficient contains zero, the overall goodness of fit (WatanabeAkaike Information Criterion, WAIC) (Watanabe, 2010) and the proportion of areas included in the groups. Smaller values of WAIC indicate a better fit.

\section{Results}

\subsection{Annual dengue cases 2002-2017}

A descriptive analysis of the number of Makassar dengue cases from 2002 to 2017 are presented in Supplementary Table S1 and Fig. 2. Spearman correlations between dengue cases and climatic covariates are given in Table 1 . It is clearly seen that there was a negative correlation between rainfall and dengue, and between average temperature and dengue, whereas average humidity was positively correlated. Maximum temperature and minimum temperature were not significantly correlated with annual dengue cases.

The districts included in each group under the ST CAR localised model with $G=2$ and $G=3$ without covariates are given in supplementary Table S2. Both models support two groups. Interestingly, under the ST CAR localised model with $G=3$, only one group was identified from 2008 to 2017. Table 2 displays the WAIC, and number of data points for each group with and without climatic covariates under the ST CAR localised model with $G=2$ and $G=3$.

Overall, the best model based on annual dengue data was an ST CAR localised model with three groups and incorporation of average humidity as it fulfilled all three metrics: the 95\% posterior credible interval $(\mathrm{CI})$ for the humidity coefficient does not contain zero; it has smaller WAIC (Watanabe, 2010), and there is at least one observation in each group. Under this model, there was one data point assigned to the first group, and 171 and 52 data points assigned to the second and the third groups, respectively. The posterior distribution for the coefficient of average humidity had a median of 0.17 (95\% CI: 0.03 , $0.26)$.

A visualisation of the grouping structures with and without covariates from 2002 to 2017 is presented in Fig. 3. This figure shows how the group structures changed with the inclusion of covariates for all areas and over the time period.

From Fig. 3, it can be clearly seen that the CAR ST localised model with $G=3$ and incorporation of average humidity can fit the data better than a corresponding model without covariates. Fig. 3(a) for the model without covariates depicts two groups whereas Fig. 3(b) for the model with average humidity as a covariate depicts three groups, with one group (Group 1) containing one district in one year (2014). An appealing feature of this CAR ST localised model with incorporation of average humidity is the ability to identify outliers as discussed below. For instance, in 2009, the ST CAR localised model without covariates consists of only one group while the ST CAR localised model with covariates consists of two groups, with one district (Rappocini) separated from the other districts. The number of dengue cases in the Rappocini district (68 cases) is substantially higher than other districts (ranging from 3 to 38 cases). Another example is evident in 2014, with the ST CAR localised model without covariates supporting only one group, while the ST CAR localised model with covariates comprises two groups (Ujung Tanah district with no cases) separated from the other districts (dengue counts ranging from 2 to 41). Details of the preferred ST CAR localised models with and without covariates as well as the fitted values are presented in supplementary Table S3.

\subsection{Monthly dengue cases 2013-2017}

Spearman correlations between dengue cases and monthly climatic covariates are given in Table 3. It is clearly seen the average humidity was positively correlated with monthly dengue cases, whereas rainfall, average temperature, maximum temperature, and minimum temperature were not significant at the 5\% level. 


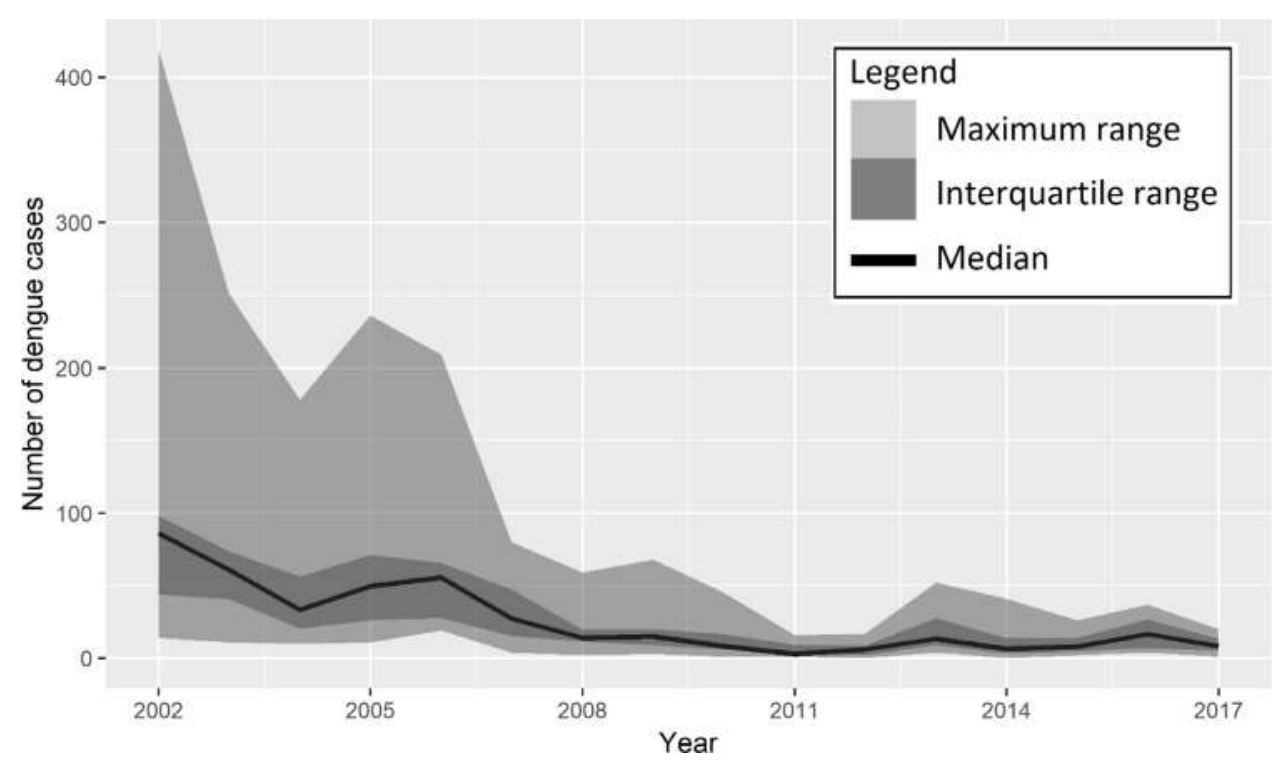

Fig. 2. Number of dengue cases across 14 districts of Makassar, Indonesia, 2002-2017.

Table 1

Correlation between dengue and climatic covariates for annual data 2002-2017.

\begin{tabular}{lllllll}
\hline & Dengue & Rainfall & Av.Temp & Max.Temp & Min.Temp & Av.hum \\
\hline Rainfall & -0.225 & & & & \\
& $(0.001)$ & & & & \\
Av.Temp & -0.322 & 0.184 & & & \\
& $(0.000)$ & $(0.006)$ & & & \\
Max.Temp & -0.116 & -0.237 & 0.642 & & \\
& $(0.084)$ & $(0.000)$ & $(0.000)$ & & \\
Min.Temp & 0.033 & 0.239 & 0.704 & 0.382 & \\
Av.hum & $(0.622)$ & $(0.000)$ & $(0.000)$ & $(0.000)$ & -0.078 \\
& 0.189 & 0.293 & -0.331 & -0.695 & $(0.000)$ & $(0.245)$ \\
\hline
\end{tabular}

Cell Contents: Spearman rho.

( $p$-value)

Table 2

Bayesian ST CAR Localised for $G=2$ an $G=3$ with and without climatic covariates for annual dengue cases 2002-2017.

\begin{tabular}{|c|c|c|c|c|c|c|c|c|c|}
\hline & \multicolumn{4}{|c|}{ ST CAR Localised with $G=2$} & \multicolumn{5}{|c|}{ ST CAR Localised with $G=3$} \\
\hline & Models & WAIC & G1 & G2 & Models & WAIC & G1 & G2 & G3 \\
\hline 1 & Without Covariates & 2045.3 & 134 & 90 & Without Covariates & 1915.2 & 0 & 165 & 59 \\
\hline 2 & $\mathrm{R}^{*}+\mathrm{AT}^{*}+\mathrm{AH}$ & 3613.3 & 223 & 1 & $\mathrm{R}^{*}+\mathrm{AT}^{*}+\mathrm{AH}^{*}$ & 1979.8 & 1 & 180 & 43 \\
\hline 3 & $\mathrm{R}^{*}+\mathrm{AH}^{*}$ & 1809.7 & 224 & 0 & $R+\mathrm{AH}^{*}$ & 1758.4 & 0 & 166 & 58 \\
\hline 4 & $\mathrm{R}$ & 2081.7 & 139 & 85 & $\mathrm{R}$ & 1880.2 & 0 & 165 & 59 \\
\hline 5 & $\mathrm{AH}^{*}$ & 1828.3 & 149 & 75 & $\mathrm{AH}^{*}$ & 1837.8 & 1 & 171 & 52 \\
\hline 6 & AT & 2251.0 & 139 & 85 & $\mathrm{AT}^{*}$ & 1862.0 & 1 & 179 & 44 \\
\hline 7 & $\mathrm{R}+\mathrm{AT}^{*}$ & 2431.4 & 140 & 84 & $\mathrm{R}^{*}+\mathrm{AT}^{*}$ & 1878.4 & 0 & 182 & 42 \\
\hline 8 & $\mathrm{R}+\operatorname{MinT}$ & 2412.2 & 138 & 86 & $\mathrm{R}+\mathrm{MinT}^{*}$ & 2178.1 & 1 & 184 & 39 \\
\hline 9 & $\mathrm{R}+\mathrm{MaxT}^{*}$ & 2069.6 & 136 & 88 & $\mathrm{R}+\mathrm{MaxT}^{*}$ & 1731.0 & 1 & 182 & 41 \\
\hline
\end{tabular}

The values in columns denoted G1, G2, G3 are the number of observations in groups one, two, and three, respectively.

$\mathrm{R}, \mathrm{AT}, \mathrm{AH}, \mathrm{MinT}$, MaxT are rainfall, average temperature, average humidity, minimum temperature and maximum temperature, respectively.

* $95 \%$ posterior credible interval $(\mathrm{CI})$ for the coefficient does not contain zero.

An ST CAR localised model with $G=2$ and $G=3$ for monthly dengue cases in 2013-2017 with climatic covariates at a temporal lag of 0 or 1 month were also analysed (see supplementary Tables S4 and S5 respectively). The results showed that all model combinations consist of one group only. Average humidity is the only climatic covariate that is substantive for a lag of 0 months, while no climatic covariates were substantive for a lag of 1 month.

As the ST CAR localised model with $G=2$ and $G=3$ for monthly dengue cases and all combinations of models with and without climatic covariates (lag 0 and lag 1 ) consist of one group only, the ST CAR AR was used to analyse these monthly dengue data. Table 4 shows the WAIC under the ST CAR AR model with and without climatic covariates. The posterior median estimate for rainfall was $-0.2676(95 \% \mathrm{CI}:-0.4698,-0.0580)$ and for average humidity was 0.42 (95\% Cl: $0.17,0.65)$.

Under the ST CAR AR model (see Table 4), rainfall and average humidity were the climatic covariates that were substantively associated with the number of dengue cases and this model had 


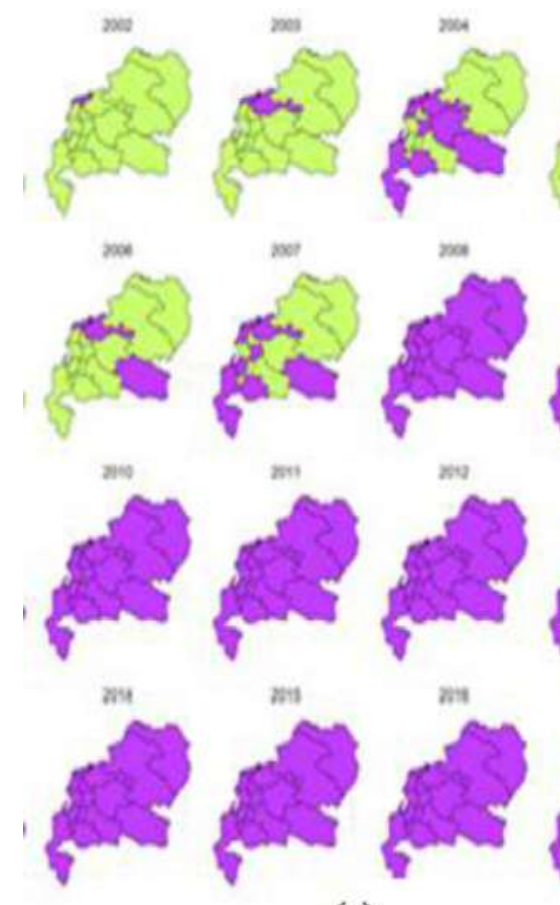

(a)
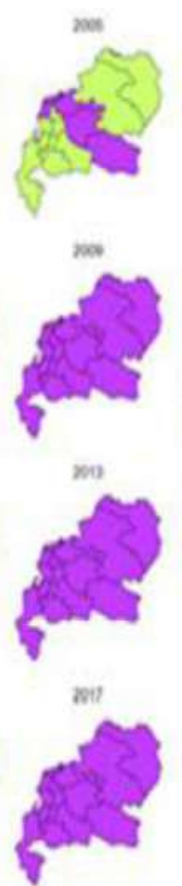

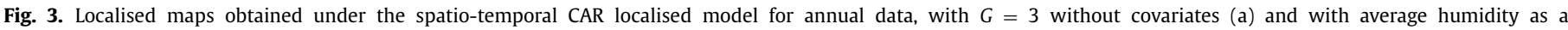
covariate (b).

Table 3

Correlation between dengue and climatic covariates for monthly data January 2013 - December 2017.

\begin{tabular}{lllllll}
\hline & Dengue & Rainfall & Av.Temp & Max.Temp & Min.Temp & Av.Hum \\
\hline Rainfall & 0.035 & & & & \\
& $(0.308)$ & & & & \\
Av.Temp & -0.008 & -0.408 & & & \\
& $(0.821)$ & $(0.000)$ & & & \\
Max.Temp & -0.022 & -0.647 & 0.887 & & \\
& $(0.518)$ & $(0.000)$ & $(0.000)$ & & \\
Min.Temp & 0.045 & 0.144 & 0.649 & 0.464 & \\
Av.hum & $(0.189)$ & $(0.000)$ & $(0.000)$ & $(0.000)$ & 0.127 \\
& 0.125 & 0.889 & -0.480 & -0.693 & $(0.000)$ \\
\hline
\end{tabular}

Cell Contents: Spearman rho.

( $p$-value).

the smallest WAIC value. In conclusion, for monthly data, from all model combinations (ST CAR localised model $G=2$ and $G=3$ without covariates and with covariates (lag 0 and lag 1), and ST CAR AR model), an ST CAR AR with the incorporation of rainfall and average humidity was preferred.

\section{Discussion}

A number of previous studies have explored the relationships between climatic covariates and dengue fever (Arcari et al., 2007; Cheong et al., 2013; Johansson et al., 2009; Karim et al., 2012). For instance, Sani et al. (2015) used a Bayesian spatial-temporal model and found that both rainfall and population density increased the risk of dengue in Kendari, Southeast Sulawesi, Indonesia. Most previous studies have used an intrinsic CAR prior structure for spatial and temporal variation to model dengue fever (Aswi et al., 2019), although more recent papers have also explored the use of the Leroux prior (Martínez-Bello et al., 2017, 2018). The Leroux CAR prior model has also been compared with BYM CAR model in modelling dengue fever (Martínez-Bello et al., 2017). However, none these models allowed for discontinuities in risk between neighbouring areas. The relatively recent ST CAR localised model allows for spa-
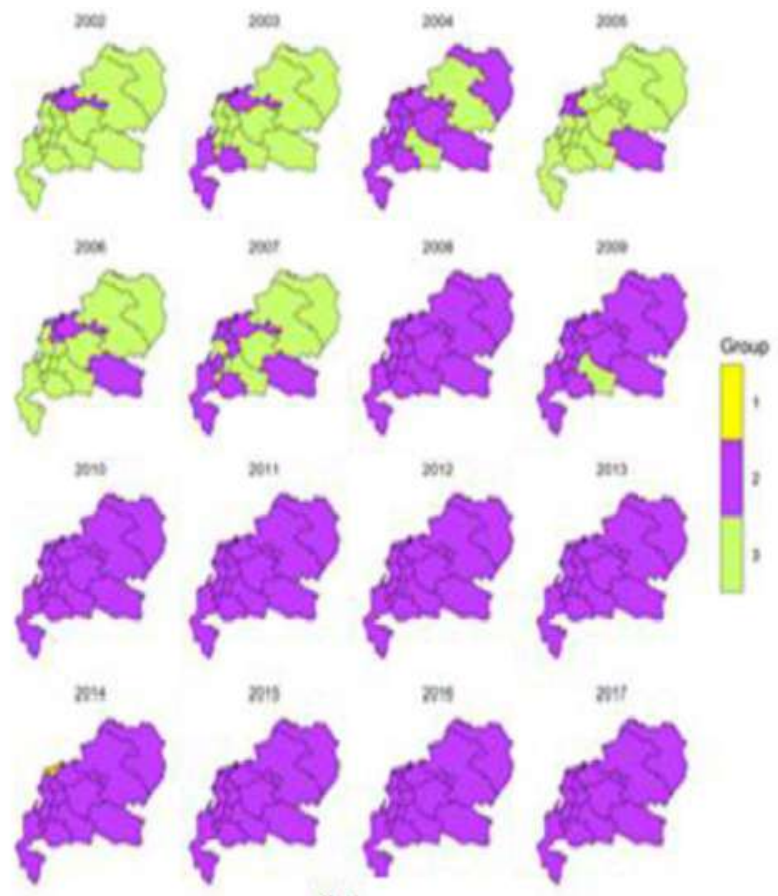
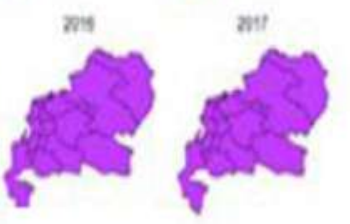

(b)

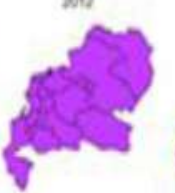

$(0.000)$
Table 4

Bayesian ST CAR AR model with and without climatic covariates for monthly dengue cases 2013-2017.

\begin{tabular}{lllll}
\hline Models & WAIC & rho.S & rho.T \\
\hline 1 Without covariates & 2037.75 & 0.55 & 0.59 \\
2 & Rainfall* + average temperature + average humidity* $^{*} 2010.17$ & 0.35 & 0.54 \\
3 & Rainfall* + average humidity $^{*}$ & 2013.13 & 0.35 & 0.54 \\
4 & Rainfall $^{*}$ & 2028.54 & 0.55 & 0.59 \\
5 & Average humidity* & 2061.28 & 0.47 & 0.56 \\
6 & Average temperature & 2079.45 & 0.52 & 0.57 \\
7 & Rainfall + average temperature & 2019.18 & 0.55 & 0.59 \\
8 & Rainfall + minimum temperature & 2020.13 & 0.57 & 0.59 \\
9 & Rainfall + maximum temperature & 2031.78 & 0.55 & 0.59 \\
\hline
\end{tabular}

rho.S and rho.T measure spatial and temporal autocorrelation, respectively.

* $95 \%$ posterior credible interval $(\mathrm{CI})$ for the coefficient does not contain zero.

tial autocorrelation between adjacent areas within discontinuous groups (Lee and Lawson, 2016). These models are appealing since disease risks in complex urban areas tend to reveal more localised spatial structure, both for areas where they vary smoothly over space and areas where there are large changes (discontinuities) in the values (Lee and Mitchell, 2012). 
However, such complexity may not be warranted in all cases and it might be preferable to describe the data as a single group with more simple spatial and/or temporal patterns. To our knowledge, this study is the first to examine the interplay between groups, climatic covariates and priors in Bayesian spatio-temporal models of dengue fever incidence. We provide the first report of a Bayesian ST CAR localised model as well as a Bayesian ST CAR AR model for modelling the risk of dengue fever in Makassar, Indonesia.

Our results showed that for annual data, an ST CAR localised model with $G=3$ with incorporation of average humidity provided the most suitable model based on a combination of the three metrics. Interestingly, the ST CAR localised model with $G=3$ without covariates from 2002 to 2007 revealed two groups, while from 2008 to 2017 only one group was identified (see Fig. 3 and Supplementary Table S2). The number of groups seemed to be influenced by the counts of dengue cases and the variation in these counts between districts. The number of dengue cases and the variance from 2002 to 2007 were much higher (ranging from 457 to 1467 and from 550.09 to $10,622.80$ respectively) than the number of dengue cases and the variance from 2008 to 2017 (ranging from 85 to 265 and from 24.44 to 286.06 ) (see Supplementary Table S1 and Fig. 2). The preferred model (ST CAR localised with $G=3$ with average humidity) can detect the grouping structure better than an equivalent model without covariates. For example, the grouping structure changed from one group to two groups in 2009 and 2014. In 2009, Rappocini was the only district in a separate group whereas in 2014, Ujung Tanah was the only district in a separate group (see Supplementary Table S3). The ability to identify outliers in this manner is an appealing feature of the localised model. Overall, the incorporation of average humidity as a covariate influenced the number of local groups for annual data.

For monthly data, all model combinations using an ST CAR localised model with $G=2$ and $G=3$ with and without covariates consisted of one group only. At this finer temporal resolution, the numbers of cases are quite similar (ranging from 0 to 39 cases) across areas and time periods. An alternative ST CAR AR model was therefore also considered for the monthly dengue dataset. This model with incorporation of rainfall and average humidity provided the best fit based on the three metrics considered.

Overall, the ST CAR localised model seems to be a better choice when there is a relatively large contrast in the magnitude and variation in counts over districts and time periods, as seen in the annual data. In this case, data more readily separate into different groups. However, when the count data and variance are quite similar over spatial areas and time periods, the localised model is less well supported as the data tend to be classified into only a single group, so choosing a simpler model that also incorporates unstructured smoothing (such as the CAR AR model) may have greater benefits.

Our results showed that rainfall and average humidity were important covariates that influenced the number of dengue cases at a monthly temporal resolution. Using a simple correlation analysis, Malik et al., 2017 found that the occurrence of Aedes aegypti as the vector of dengue was strongly influenced by climatic factors (temperature, rainfall and humidity), and rainfall as well as humidity were dominant in their association with vector breeding. This importance of rainfall is in agreement with some other studies, but the direction of our association (negative for monthly data) was surprising. Using a Bayesian method, Lowe et al., 2016 also reported a result somewhat similar to that found in our study that there was a negative correlation between precipitation 4-6 months previously and the relative risk of dengue. Another study in Indonesia examined the relationship between five climatic factors (rainfall anomalies, rainfall, humidity, temperature, and Southern Oscillation Index (SOI)) and provincial-scale dengue/dengue haem- orrhagic fever (DHF) for eight chosen provinces using the combination of multiple regression and Pearson correlation analysis and found an inconsistent relationship between dengue incidence and climatic factors across the eight provinces (Arcari et al., 2007). They found that rainfall anomalies, rainfall and humidity exhibited both negative and positive correlations across eight provinces. One possible reason for this is that heavy precipitation/rainfall can wash out mosquito breeding sites (Thomson and Mason, 2019). This may be occurring in Makassar, but our result warrants further investigation.

The strength of the preferred model (ST CAR localised with $G=3$ with average humidity) is that it can differentiate between groups of areas that display elevated values as well as areas that display reduced values of the response. Rappocini district was identified as an outlier area with a high risk of dengue disease, while in the same manner Ujung Tanah was identified as having a low risk of dengue disease. Rappocini is a suburban district in Makassar city and has a large number of slum areas. A World Health Organisation (WHO) report on dengue prevention and control in the South-East Asia region stated that the density of Aedes aegypti is higher in suburban areas than in urban areas in Indonesia due to the traditional water storage practices. Furthermore, the premise index for Aedes aegypti was the highest in slum areas houses (Plianbangchang, 2011). This may explain in part the results of our study.

However, our results are limited by not having data on all covariates for each of the spatial areas at each time period. This was understandable in this case study as Makassar covers a small area $\left(175.79 \mathrm{~km}^{2}\right.$ ) and has a small number of areas (14 administrative districts). A more complete dataset may affect the results. Additionally, for dengue data the greatest temporal resolution available was monthly. Given the incubation period is much less than 1 month within mosquitos (8 to 12 days), and humans ( 3 to 14 days), having daily or weekly data would have been preferable to examine climatic influences.

\section{Conclusions}

In summary, our results suggest that using appropriate Bayesian spatio-temporal models enables identification of different groups of areas and the impact of climatic covariates. Detection of specific high-risk areas and of the interplay between these areas, climatic variables and the temporal resolution of the data, is beneficial for the policy maker as it may help in decision making.

\section{Acknowledgements}

The authors acknowledge the City Health Department of Makassar, South Sulawesi Province for providing the dengue fever datasets and the Meteorology, Climatology, and Geophysical Agency for providing climatic covariates.

\section{Funding}

This work was supported by the ARC Centre of Excellence for Mathematical and Statistical Frontiers (ACEMS), project ID CE140100049 and Queensland University of Technology (QUT). The authors reported no other financial interests related to this research.

\section{Supplementary materials}

Supplementary material associated with this article can be found, in the online version, at doi:10.1016/j.sste.2020.100335. 


\section{References}

Arcari, P., Tapper, N., Pfueller, S., 2007. Regional variability in relationships between climate and dengue/DHF in Indonesia. Singap. J. Trop. Geogr. 28 (3), 251-272. doi:10.1111/j.1467-9493.2007.00300.x.

Aswi, A., Cramb, S.M., Moraga, P., Mengersen, K., 2019. Bayesian spatial and spatiotemporal approaches to modelling dengue fever: a systematic review. Epidemiol. Infect. 147. doi:10.1017/S0950268818002807.

Besag, J., York, J., Mollié, A., 1991. Bayesian image restoration, with two applications in spatial statistics. Ann. Inst. Stat. Math. 43 (1), 1-20.

BPS, 2018. Makassar Municipality in Figures 2018 Technical Report 1102001.7371. Makassar BK.

Chan, M., 2012. Global Strategy For Dengue Prevention and Control. WHO, Geneva, Switzerland Technical Report WC-528.

Chan, M., Johansson, M.A., 2012. The incubation periods of dengue viruses. PLoS ONE 7 (11). doi:10.1371/journal.pone.0050972.

Cheong, Y.L., Burkart, K., Leitão, P.J., Lakes, T., 2013. Assessing weather effects on dengue disease in Malaysia. Int. J. Environ. Res. Public Health 10 (12), 63196334. doi:10.3390/ijerph10126319.

Gubler, D.J., 1998. Dengue and dengue hemorrhagic fever. Clin. Microbiol. Rev. 11 (3), 480-496.

Johansson, M.A., Dominici, F., Glass, G.E., 2009. Local and global effects of climate on dengue transmission in Puerto Rico. PLoS Negl. Trop. Dis. 3 (2), e382. doi:10. 1371/journal.pntd.0000382.

Karim, M., Munshi, S., Anwar, N., 2012. Climatic factors influencing dengue cases in Dhaka city: a model for dengue prediction. Indian J. Med. Res. 136 (1), 32-39.

Knorr-Held, L., 1999. Bayesian modelling of inseparable space-time variation in disease risk. Stat. Med. 19 (17-18), 2555-2567. doi:10.1002/1097-0258.

Lee, D., Lawson, A., 2016. Quantifying the spatial inequality and temporal trends in maternal smoking rates in Glasgow. Ann. Appl. Stat. 10 (3), 1427-1446. doi:10. 1214/16-AOAS941.

Lee, D., Mitchell, R., 2012. Boundary detection in disease mapping studies. Biostatistics 13 (3), 415-426. doi:10.1093/biostatistics/kxr036.

Lee, D., Mitchell, R., 2013. Locally adaptive spatial smoothing using conditional autregressive models. J. R. Stat. Soc. Ser. C Appl. Stat. 62 (4), 593-608. doi:10.1111/ rssc.12009.

Lee, D., Sarran, C., 2015. Controlling for unmeasured confounding and spatial misalignment in long-term air pollution and health studies. Environmetrics 26 (7), 477-487.

Leroux, B.G. Lei, X., Breslow, N., 2000. Estimation of disease rates in small areas: a new mixed model for spatial dependence. In: Halloran, M.E., Berry, D. (Eds.), Statistical Models in epidemiology, the environment, and Clinical Trials. Springer-Verlag, New York, pp. 179-191.
Lowe, R., Cazelles, B., Paul, R., Rodó, X., 2016. Quantifying the added value of climate information in a spatio-temporal dengue model. Stoch. Environ. Res. Risk Assess. 30 (8), 2067-2078. doi:10.1007/s00477-015-1053-1.

Malik, A., Yasar, A., Tabinda, A.B., Zaheer, I.E., Malik, K., Batool, A., et al., 2017. Assessing spatio-temporal trend of vector breeding and dengue fever incidence in association with meteorological conditions. Environ. Monit. Assess. 189 (4), 1 20. doi:10.1007/s10661-017-5902-X.

Martínez-Bello, D., López-Quílez, A., Prieto, A., 2017. Relative risk estimation of dengue disease at small spatial scale. Int. J. Health Geogr. 16 (1), 1-15. doi:10. 1186/s12942-017-0104-X.

Martínez-Bello, D., López-Quílez, A., Prieto, A., 2018. Spatiotemporal modeling of relative risk of dengue disease in Colombia. Stoch. Environ. Res. Risk Assess. 32 (6) 1587-1601. doi:10.1007/s00477-017-1461-5.

Plianbangchang, S., 2011. Comprehensive Guidelines For Prevention and Control of Dengue and Dengue Haemorrhagic Fever. WHO, India Technical Report WC 528

R Core Team, 2019. R: A language and Environment For Statistical Computing. R Foundation for Statistical Computing, Vienna, Austria Retrieved from http: /www.R-project.org/

Rodrigues, E.C., Assunção, R., 2012. Bayesian spatial models with a mixture neighborhood structure. J. Multivar. Anal. 109 (C), 88-102. doi:10.1016/j.jmva.2012.02. 017.

Rushworth, A., Lee, D., Mitchell, R., 2014. A spatio-temporal model for estimating the long-term effects of air pollution on respiratory hospital admissions in Greater London. Spat. Spatiotemporal Epidemiol. 10, 29-38. doi:10.1016/j.sste. 2014.05.001.

Sani, A., Abapihi, B., Mukhsar, M., Kadir, K., 2015. Relative risk analysis of dengue cases using convolution extended into spatio-temporal model. J. Appl. Stat. 42 (11), 2509-2519. doi:10.1080/02664763.2015.1043863.

Thomson, M.C., Mason, S.J., 2019. Climate Information For Public Health Action, 1st ed. Routledge, London.

Vanessa, R., Rebecca, R., Shilu, T., Wenbiao, H., 2012. Surveillance of dengue fever virus: a review of epidemiological models and early warning systems. PLoS Negl. Trop. Dis. 6 (5), e1648.

Watanabe, S., 2010. Asymptotic equivalence of bayes cross validation and widely applicable information criterion in singular learning theory. J. Mach. Learn. Res. 11, 3571-3594.

WHO-SEARO, 2011. Reported Cases of Dengue and Deaths from the SEA Countries 2000-2011 Retrieved from http://www.searo.who.int/entity/vector_borne tropical_diseases/data/ReportedCasesDeaths.pdf

Yu, H.L., Lee, C.H., Chien, L.C., 2016. A spatiotemporal dengue fever early warning model accounting for nonlinear associations with hydrological factors: a Bayesian maximum entropy approach. Stoch. Environ. Res. Risk Assess. 30 (8), 2127-2141. doi:10.1007/s00477-016-1328-1. 